\title{
Parents' Perspectives on Variants of Uncertain Significance from Chromosome Microarray Analysis
}

\author{
Lesli A. Kiedrowski ${ }^{1,2} \cdot$ Kailey M. Owens ${ }^{3} \cdot{\text { Beverly M. } \text { Yashar }^{2} \text { - Jane L. Schuette }}^{3}$
}

Received: 18 December 2014 / Accepted: 12 May 2015 /Published online: 19 May 2015

(C) National Society of Genetic Counselors, Inc. 2015

\begin{abstract}
Chromosomal microarray analysis (CMA) for unexplained anomalies and developmental delay has improved diagnosis rates, but results classified as variants of uncertain significance (VUS) may challenge both clinicians and families. We explored the impact of such results on families, including parental knowledge, understanding and interpretation. Semi-structured telephone interviews were conducted with parents $(N=14)$ who received genetic counseling for a VUS in their child. Transcripts were analyzed through an iterative coding process. Participants demonstrated a range of recall and personal interpretation regarding whether test results provided a causal explanation for their children's health issues. Participants maintained contradictory interpretations, describing results as answers while maintaining that little clarification of their child's condition had been provided. Reported benefits included obtaining medical services and personal validation. Parents described adaptation/coping processes similar to those occurring after positive test results. Recall of terminology, including "VUS" and precise CMA abnormalities, was poor. However, most demonstrated conceptual understanding of scientific uncertainty. All participants expressed intentions to return for recommended genetics follow-up but had misconceptions about how this would occur. These results provide
\end{abstract}

Lesli A. Kiedrowski

lesli.kiedrowski@utsouthwestern.edu

1 Department of Cancer Genetics, University of Texas Southwestern Medical Center, 5323 Harry Hines Blvd, Dallas, TX 75390-9323, USA

2 Department of Human Genetics, University of Michigan, Ann Arbor, Michigan, USA

3 Department of Pediatrics and Communicable Diseases, University of Michigan, Ann Arbor, Michigan, USA insight into the patient-and-family experience when receiving uncertain genomic findings, emphasize the importance of exploring uncertainty during the communication process, and highlight areas for potential attention or improvement in the clinical encounter.

Keywords Genetic counseling - Chromosomal microarray · Uncertainty $\cdot$ Genetic testing $\cdot$ Qualitative

\section{Introduction}

Chromosomal microarray analysis (CMA) to detect copy number variations (CNVs) is used as a first-tier diagnostic genetic test for individuals with unexplained congenital anomalies or developmental disabilities (Manning et al. 2010; Miller et al. 2010a). The identification of a genetic disorder can provide information to families and care providers regarding prognosis, medical management, and reproductive decisionmaking (Lopez-Rangel et al. 2008). In well-defined chromosomal deletion/duplication syndromes, clinical management may include referrals to other health care providers based on risks for associated medical complications and follow-up in genetics clinic at recommended intervals. In the absence of such a diagnosis, determining appropriate follow-up and management may be more challenging.

Due to large gaps in knowledge of the phenotypic effects of many CNVs, the interpretation of array results can be problematic (Coughlin et al. 2012). Given their novelty and lack of functional data for the majority of genes, it is not uncommon for CMA results to be classified as variants of uncertain clinical significance (VUS) (Lee et al. 2007). In some cases, parental testing may help to clarify whether a $\mathrm{CNV}$ is benign or pathogenic (Shaffer et al. 2008), but if parental samples are unavailable or uninformative and there is limited description 
of the CNV in the medical literature, interpretation of results is often consistent with a VUS (Manning et al. 2010). This "genetic ambiguity" can be difficult for families to process. They are confronted with a genetic test result that may not provide an explanation for their child's condition, leaving them with uncertainty regarding a diagnosis.

There is a wide body of literature, spanning several decades, surrounding diagnostic uncertainty in pediatric illness. The vast majority focuses on parental attitudes, stress, coping, and adaptation to a lack of diagnosis for their ill children (Lipinski et al. 2006; Reiff et al. 2012; Whitmarsh et al. 2007). While such studies are relevant to the provision of genetic counseling for results of uncertain significance, they focus on parents' psychological response to genetic diagnoses, or lack thereof, rather than their understanding of an inherently uncertain finding.

Uncertainty in the setting of a genetic diagnosis is a more recent area of exploration (Lipinski et al. 2006; Whitmarsh et al. 2007), although little research has been conducted to determine how parents interpret and utilize a VUS. Since parents have certain expectations as to what a genetic diagnosis will mean for their family, such as the anticipation of an explanation (Lewis et al. 2010; Michie et al. 1997) and improved medical management (Graungaard and Skov 2007; Lewis et al. 2010; Lopez-Rangel et al. 2008; Michie et al. 1997; Rosenthal et al. 2001), exploring uncertainty in this context may help to ensure better understanding and adaptation to ambiguity. A study exploring parental response to CMA results identified themes of incomplete comprehension and struggles to derive personal meaning among parents whose children had received a pathogenic or a VUS CMA result (Reiff et al. 2012). When examining preferences for results from microarrays, Turbitt et al. (2014) found respondents favored knowing about variants for their potential practical benefits, though they also agreed that such results would raise parental anxiety (Turbitt et al. 2014). A recent study assessed parental understanding, perceived value, perceptions of child vulnerability, and parental stress after receiving a VUS (Jez et al. 2015). While findings were consistent with ongoing difficulties with ambiguity, perceptions that the VUS had provided some explanation and relief were also noted. Questions still remain about the parental effort to adapt to and cope with the diagnostic ambiguity of a VUS.

\section{Purpose of the Study}

The purpose of this exploratory qualitative study was to further investigate the interpretation and impact of genetic test results of uncertain significance in the context of pediatric chromosomal microarray analysis. A better understanding of patients' and families' perspectives on ambiguous test results and their impact on care management plans is crucial to providing effective genetic counseling services, especially as we enter the era of genomic sequencing (Ali-Khan et al. 2009; Fanos 2012; Miller et al. 2010b).

\section{Methods}

\section{Participants}

Families of children who underwent chromosomal microarray analysis through the Michigan Medical Genetics Laboratories (MMGL) at the University of Michigan Health System (UMHS) and received genetic counseling for a CMA VUS at the UMHS Pediatric Genetics clinic between February 2011 and March 2013 were eligible for inclusion. This timeframe was chosen in order to minimize recall bias while still providing a large enough sample for recruitment. During this period, the Pediatric Genetics clinic was staffed by up to three genetic counselors and six pediatric geneticists. Families entered the CMA counseling session as either established patients of the Pediatric Genetics clinic, in which case testing was ordered by a Pediatric Genetics provider, or as new patients, in which case testing had been ordered by another subspecialist at UMHS. For established Pediatric Genetics patients, initial results disclosure usually occurred prior to the counseling session in the form of a phone conversation. For new patients, there was a variety of disclosure formats, e.g., phone or in-person conversation with a non-geneticist. A typical CMA counseling session included a discussion between the child's parent(s), one of the genetic counselors, and/or one of the geneticists. Topics discussed included the CMA VUS, its implications, and recommended follow-up evaluations. A letter summarizing this discussion was sent to both the family and the referring physician following the appointment for both new and returning patients.

\section{Instrumentation}

A semi-structured interview guide was developed specifically for this study based on available literature in the subject area, supplemented by anecdotal experience of clinical genetic counselors. Open-ended questions with follow-up prompts assessed parental recall of the test result, personal understanding and interpreted meaning(s) of the test result, emotional response and adjustment to receiving the test result, interpretation of parental test results (if performed), motivation to follow up with recommended return visits to the genetics clinic and other referrals, and reflections on the helpfulness of genetic counseling (see example questions in Table 1). This interview guide was piloted with pediatric genetic counselors. It was not piloted with parents from the target study population, given the knowledge that the population was small and concerns regarding response rate and expected sample size of study interviews. 
Table 1 Condensed Codebook ${ }^{\mathrm{a}}$ with Example Questions from Interview Guide

\begin{tabular}{|c|c|c|c|}
\hline Domain $^{b}$ & Thematic content areas & Sub-themes & Example questions \\
\hline & \multirow[t]{3}{*}{ Pre-CMA testing } & & $\begin{array}{l}\text { How did you and your child end up } \\
\text { in the genetics clinic? }\end{array}$ \\
\hline & & Coping & $\begin{array}{l}\text { How important was it to you to have } \\
\text { a diagnosis for your child's health issues? }\end{array}$ \\
\hline & & Diagnostic odyssey & \\
\hline \multirow[t]{3}{*}{ Parental Recall \& Understanding } & Comprehension of CMA & & Do you recall what this test was looking for? \\
\hline & & How testing works & \\
\hline & & Reason for testing & \\
\hline \multirow[t]{6}{*}{ Parental Recall \& Understanding } & Comprehension of results & & \\
\hline & & Recall of result & Do you recall the result of the test? \\
\hline & & Self-rated understanding & $\begin{array}{l}\text { Can you tell me more about your own } \\
\text { personal understanding of the result? }\end{array}$ \\
\hline & & Self-rated genetics knowledge & \\
\hline & & Recall of VUS terminology & $\begin{array}{l}\text { Do you remember the counselor or doctor } \\
\text { discussing uncertain significance? }\end{array}$ \\
\hline & & Scientific uncertainty & \\
\hline \multirow[t]{4}{*}{ Impact of results } & Impact of results & & Did this information change anything for you? \\
\hline & & Access to services & \\
\hline & & Information & \\
\hline & & Emotional impact & $\begin{array}{l}\text { What emotions do you remember experiencing } \\
\text { when you received/discussed the test result? }\end{array}$ \\
\hline \multirow[t]{6}{*}{ Impact of results } & Coping & & $\begin{array}{l}\text { Did the information change your ability to deal } \\
\text { with your child's health? }\end{array}$ \\
\hline & & Empowerment & \\
\hline & & Engagement & \\
\hline & & Acceptance & \\
\hline & & Optimism/Pessimism & \\
\hline & & Family support & \\
\hline \multirow[t]{6}{*}{ Parental interpretation } & Interpretation of result & & $\begin{array}{l}\text { Do you feel that the genetic change found } \\
\text { through this testing explains } \\
\text { your child's health issues? }\end{array}$ \\
\hline & & Provides an answer & \\
\hline & & Is the cause & \\
\hline & & Meaning not clear & \\
\hline & & Lack of resolution & \\
\hline & & Limits of prognostic information & $\begin{array}{l}\text { What does this result say to you about } \\
\text { your child's future? }\end{array}$ \\
\hline \multirow[t]{9}{*}{ Genetics Follow-up } & Follow-up in genetics & & $\begin{array}{l}\text { What role will the genetics clinic play in your } \\
\text { child's medical care going forward? }\end{array}$ \\
\hline & & Motivation & $\begin{array}{l}\text { Do you plan to go back to the genetics } \\
\text { clinic in the future? }\end{array}$ \\
\hline & & Anticipation of future information & \\
\hline & & Ongoing provider expertise & \\
\hline & & Understanding of reason for follow-up & \\
\hline & Patient satisfaction & & \\
\hline & & Staff communication & \\
\hline & & Staff supportiveness & \\
\hline & & Suggestions for improvement & \\
\hline
\end{tabular}

${ }^{\mathrm{a}}$ Individual codes $N=130$

${ }^{\mathrm{b}}$ Thematic content areas not assigned to a domain are not presented in this paper 


\section{Procedures}

Parents of children who received genetic counseling for a CMA VUS were identified by review of a database maintained by MMGL and mailed an invitation to participate in the study as well as a mail-back response card to indicate interest. Additionally, from October-December 2012, parents were recruited prospectively during the CMA VUS counseling session; in these cases, at the end of the counseling session, parents were provided with a copy of the same invitation materials and response card. Only one parent of each child was eligible to participate. There were no other inclusion or exclusion criteria specified for participation. Participants were offered a \$15 gift card in appreciation of their time. This study was approved by the Institutional Review Board at the University of Michigan Medical School (HUM\#00062486).

Semi-structured interviews were conducted over the telephone by one author (L.A.K.). The author conducting the interview was not part of the clinical care team for any of the participants. Some of the study team had provided clinical genetic counseling to the families of parents interviewed; however, each interview and corresponding transcript was assigned a numeric identifier. Personal identifiers were removed from the transcripts in an effort to conceal the identity of the interview subject to the study team when reviewing the data.

\section{Data Analysis}

Interviews were recorded and transcribed verbatim. Each interview was linked to the specific VUS result(s) for the child. Transcripts were analyzed for cross-cutting themes through an iterative group coding process (DeCuir-Gunby et al. 2011). The authors met on a weekly or bi-weekly basis to review coding progress, compare coded samples, and work toward consensus. Half of the transcripts (7/14) were coded and discussed by all authors to identify and resolve coding discrepancies and for codebook revisions. Upon reaching full group consensus on these transcripts, one author (K.M.O.) independently re-coded the seven initial transcripts using the final version of the codebook. One author (L.A.K.) independently coded the remaining transcripts and reviewed them with the other authors to ensure that full group agreement was achieved.

Many thematic content areas (domains) were identified in the transcripts (Table 1). We chose to highlight 4 domains having relevance for counseling for genomic testing and selected 21 quotations from 8 of the 14 participants which were felt to be illustrative for presentation. Other quotations, although usually consistent with the selections, were not chosen for a variety of reasons, such as length, clarity, and/or fluency.

\section{Results}

\section{Participant Characteristics}

Parents of 55 children were identified as eligible and invited to participate. Fourteen parents representing 14 different families were interviewed (participation rate $25 \%$ ). Interviews ranged in length from 23 to $77 \mathrm{~min}$ (average $48 \mathrm{~min}$ ). The participants included parents of patients with a wide range of indications for genetic testing, including multiple congenital anomalies, developmental delay/autism, behavioral concerns, and chronic medical conditions. The majority of parents were Caucasian females who had completed at least a high school education (Table 2). The average time between results disclosure and phone interviews was 6.4 months (range 1-15 months).

Nine of 14 participants' children underwent CMA after meeting with a member of the pediatric genetics team. The remaining 5 participants had testing ordered by the UMHS Division of Pediatric Neurology and were referred for genetic counseling after an abnormality was detected.

\section{Themes Identified in Interview Responses}

The number of responses coded as consistent with a particular theme are provided in parentheses below. It is important to note that the numbers do not always equal the total sample size due to contradictory statements made by participants.

\section{Parental Recall and Understanding}

Parental comprehension of the testing process and results was explored by assessing recall and eliciting participants' selfrated understanding. Several parents could not recall the purpose of testing, stating that they proceeded with testing because a physician requested it $(n=8)$. Others considered the reason for testing to be "to rule out medical problems" and did not specifically mention chromosomes or genetic conditions. Some felt confident in their self-rated comprehension prior to receiving results $(n=5)$, while others reported that they had failed to grasp the details of testing ( $n=3$ with partial understanding, $n=5$ with absent understanding). For some, this related to a self-reported lack of genetic literacy. Most parents could not specify that CMA identifies CNVs; some thought that the test involved DNA sequencing.

While 3 of 14 parents were able to report their child's CMA result accurately, including specific chromosomal bands, most only recalled that there was extra or missing material; 7 remembered which chromosome, while 1 reported an incorrect chromosome and 3 did not remember. Another mother incorrectly reported the results of parental testing as negative when, in fact, the variant had been maternally inherited.

Similarly, participants' self-rated understanding of test results varied widely between complete $(n=3)$, partial $(n=6)$, 
Table 2 Characteristics of Participants $(N=14)$

\begin{tabular}{|c|c|c|}
\hline & Number & Percentage $^{a}$ \\
\hline \multicolumn{3}{|l|}{ Patient's Age } \\
\hline $3-4$ years & 4 & 29 \\
\hline $5-10$ years & 7 & 50 \\
\hline $11-19$ years & 3 & 21 \\
\hline \multicolumn{3}{|l|}{ Patient's Gender } \\
\hline Female & 7 & 50 \\
\hline Male & 7 & 50 \\
\hline \multicolumn{3}{|l|}{ CNV Inheritance ${ }^{b}$} \\
\hline De Novo & 2 & 13 \\
\hline Maternal & 5 & 33 \\
\hline Paternal & 1 & 7 \\
\hline Unknown & 7 & 47 \\
\hline \multicolumn{3}{|c|}{ Number of Months between Results Disclosure and Interview } \\
\hline $1-3$ & 4 & 29 \\
\hline $4-8$ & 7 & 50 \\
\hline $9-15$ & 3 & 21 \\
\hline \multicolumn{3}{|l|}{ Parent's Gender } \\
\hline Female & 13 & 93 \\
\hline Male & 1 & 7 \\
\hline \multicolumn{3}{|l|}{ Parent's Race } \\
\hline White & 11 & 79 \\
\hline Black/African American & 2 & 14 \\
\hline Native American & 1 & 7 \\
\hline \multicolumn{3}{|c|}{ Parent's Highest Level of Education Completed } \\
\hline Less than High School Diploma & 1 & 7 \\
\hline High School Diploma & 2 & 14 \\
\hline Some College & 5 & 36 \\
\hline Associate's or Technical Degree & 3 & 21 \\
\hline Bachelor's Degree & 2 & 14 \\
\hline Graduate Degree & 1 & 7 \\
\hline
\end{tabular}

${ }^{a}$ Numbers may not add up to $100 \%$ due to rounding

${ }^{b}$ Out of 15 , as one patient had two CNVs with different inheritance patterns

and absent $(n=4)$. Several reported that they did not initially comprehend results at all (e.g., at the time of phone disclosure) but understood following in-person disclosure and discussion with the doctor/genetic counselor. Others were still not confident in their grasp of test results several months after in-person appointments. When asked how she explained to others what the testing indicated, one mother responded:

"I say she's got a genetic, um... abnormality on chromosome $15 \ldots$ And they're like, what? And it doesn't really make sense to anyone, and it doesn't really make any sense to me either." (Participant 13)

Despite 12 of 14 participants reporting that they did not recall the use of the term "uncertain significance" - terminology consistently used during visits as well as in written summary letters mailed to parents - all expressed some awareness of the inherent scientific uncertainty of their child's CMA result:

“...they were also very clear that they weren't saying that [child] is the way that she is because of this extra material...." (Participant 7)

"They were just saying we might not be able, that it could or could not affect, that there's no $100 \%$ on anything...." (Participant 11)

The rarity of the findings and lack of information available regarding the particular genetic variants were factors recalled by parents, with several specifically mentioning the doctor/ genetic counselor reporting uncertainty about whether the VUS was related to their child's symptoms due to a lack of reported cases.

\section{Impact of Results}

Participants described a wide variety of emotions when first receiving or discussing CMA results. Among the most common responses noted were relief $(n=6)$ and guilt $(n=4)$, with others reporting comfort, happiness, surprise, disappointment, sadness, frustration, fear, worry, and mixed emotions. Three parents initially reported not having significant emotional reactions, but at other points in the interview discussed having emotional responses to receiving the results. One mother described how she and her husband reacted to the information after their appointment:

“It's just kind of that moment when you just can't even, you're thinking about it, and you've processed so much, and you just kind of want to sit down and have a drink and... just be sad for a few minutes. That this is... what you're dealing with, basically. Not because you're resentful, not because you're feeling sorry for yourself or anything like that... Normal kids don't have to go through this... You just wish for some normalcy for your kids, you wish for some normalcy for what they're gonna have to go through...." (Participant 7)

Some parents - regardless of whether CNVs were de novo, inherited, or unknown - expressed strong feelings of guilt about having passed on the genetic changes or having caused their child's health concerns:

"...As a parent, what did we do? What did I do during my pregnancy? ...Was there anything we could have done to have prevented this? Did we cause it?" (Participant 2) 
Some commented on the permanence of receiving genetic test results despite the uncertainty of the interpretation, revealing feelings of sadness or loss for themselves and/or their children. Others expressed pessimism, hopelessness, and feelings of failure because of the inability to find more specific information about the cause of their child's health issues. When asked about how she felt this genetic testing impacted her outlook on her child's medical care, one mother responded:

"Just kind of feeling like you're never going to have just the right answer... your kid's never going to fit into just one box. So nobody's ever going to know what to do with her. Nobody's ever going to be able to help because she doesn't - she has this abnormality, but it's not this disorder that she has or whatever." (Participant 7)

Some participants discussed the idea that having a rare genetic variant made their child unique. Some parents viewed this as isolating, noting a lack of others who understood their situation:

\begin{abstract}
"Basically what I'm trying to say is there are support groups for Down's [sic] syndrome, there are support groups for your kid being on the spectrum of autism, but there's not a support group for Xq25. There's not that many out there to communicate with the other parents, like, 'Hey, yeah, this is what we're going through, this is what seems to help us to cope on those days that our child is having a bad day." (Participant 8)
\end{abstract}

Four parents, when asked about the effects of undergoing testing, responded that nothing had changed in their child's lives (though 2 of these 4 discussed perceived benefits of testing later in the interview). Among others, two main areas of perceived benefits emerged: access to services $(n=6)$ and information for themselves, health care providers, teachers, and/or family $(n=12)$. Several parents received referrals that either identified or ruled out additional health concerns. Some were connected with speech, physical, or occupational therapists and/or multidisciplinary clinics, and a few claimed that the results had helped other outside providers make medication decisions. Some anticipated that having test results might assist with financial resources like supplemental social security income. Many commented that simply having a result, whether diagnostic or not, facilitated the consideration of more therapeutic options by health care providers and teachers:

"I don't want to say they necessarily treat him differently, but I think that now that we have the diagnosis, they're so much more open to my suggestions and my thoughts and they seem a lot more willing to [say], 'Ok, well, we'll try this therapy out...." (Participant 2)

Some parents reported a difficult emotional reaction to the uncertainty surrounding the VUS; others described the benefit of having "a point of reference" or "something to go on." Several parents whose children had developmental and/or behavioral problems reported being relieved to finally have some indication of a potential cause. This was particularly evident in parents who reported prior frustration due to a lack of support from health care providers, family, and others. When asked how their feelings about test results had changed over time, several mentioned an emerging sense of validation $(n=7)$ :

"The biggest thing to me was just a relief that I'm not crazy, I'm not a bad mom, that there was something going on with my son. And I think that the biggest thing that I have gained from seeing you guys is the relief that finally, someone, in essence, almost kind of believes me because of the trouble that we had had for the years before that" (Participant 2).

While negative coping was frequently noted, some parents indicated positive adaptation. Many were grateful that their child did not have more severe health concerns. When prompted to discuss how they had continued with their child's medical journey after receipt of their genetic test results, several parents described engagement as a coping mechanism, mentioning feelings of empowerment as they became involved in medical management or advocacy. Some mentioned joining support groups and connecting with others after receiving genetic test results. While a few were hopeful that technology would provide future options, most acknowledged that the results, despite being ambiguous, had moved them toward accepting their child's conditions:

"Let's be honest, our genetics, this diagnosis means that... really things are the way they are... This is very, very permanent. This is more about acceptance and just trying to figure out how we can help him... it's in his DNA, this isn't something that's gonna be changed." (Participant 12)

\section{Parental Interpretation}

When asked to elaborate on personal interpretations of the CMA results, many parents described struggling to derive meaning and blamed this difficulty on a lack of information. Others were conflicted and/or contradicted themselves. Recurring throughout the interviews were the perceptions of the test result as an answer $(n=3)$, not an answer $(n=2)$, or in some 
cases, both $(n=4)$. Others did not describe the results as either an answer or lack thereof, and instead used more ambiguous descriptions.

Furthermore, parents made a distinction between results as answers versus explanations for their child's medical problems. As an answer, some expressed that the variant, despite uncertainty about pathogenicity, was a first step toward a unifying diagnosis. As an explanation, parents instead voiced dismay that little clarification was provided. Prognostic uncertainty was a recurring theme. Ten parents discussed the lack of substantive information, including not knowing what to expect developmentally, whether a risk existed for associated health problems, how to provide helpful medications or therapies, or how to plan for potential long term care:

"I'm not saying they never gave me any answers. Whatever answer they can provide me, yes, they were excellent giving me that. It's just that, as a parent of any child, you want answers for what's going on with your child. And if you don't have answers, [laughter] and if they don't have answers, it's frustrating for everybody...." (Participant 8)

"Unfortunately, in our case, we didn't really get an answer, but at least we got an answer that there was something wrong genetically, but... there wasn't much information on what was wrong...." (Participant 2)

Several parents, while able to express an accurate understanding of the clinical uncertainty associated with their result, reported feeling confident that the VUS provided a causal explanation for their child's health issues $(n=7)$. Some acknowledged this discordance, admitting that they had little justification for this view other than instinct:

“...She's got some type of genetic problem, so that's the reason why she's not learning or talking or walking and doing things that a normal 3 and half year old would do. ...It's the only explanation I have, so I just have to go with it. [laughter] I don't know what else it could be. I've been to so many other doctors and nobody can find anything, and this is the only thing that they've found, so I just have to believe that this is why [she has] all these problems." (Participant 13)

Some parents associated the CMA result with some, but not all, of their child's medical problems - especially those identified subsequent to the test results - as a consequence of recommended evaluations (e.g., echocardiograms, ophthalmologic exams):

"The things we found out afterwards, that explains all those, 'cause we checked because of that information. But why is it all in one little tiny body? No, I don't know, but I don't really know that anybody does." (Participant 11)

Those who did not interpret the CNV as an answer sometimes justified this by citing a lack of information. For example, when asked if it explained her child's health issues, one mother responded:

"Oh no, it didn't. ...Because there were no solutions.

There wasn't anything definite." (Participant 6)

Some parents had developed other causal explanations for their child's conditions, including those that were idiopathic or of an environmental etiology, and therefore had dismissed the CMA result as irrelevant:

"I really don't feel like how he speaks has anything to do with the chromosome duplication; I do believe that has to do with his ears." (Participant 12)

Two parents viewed the CMA result as providing helpful prognostic information but did not provide detail about what specifically was helpful. One parent voiced both perspectives; the results had yielded helpful prognostic information and, later, lacked such information:

"It did impact his health, now that we know what to look out for, what to watch out for and how to work with him to benefit his life a lot better because we got these results." (Participant 8)

However, later in the interview, the same mother said:

"You don't know what's going to happen the next day, because there's nothing to fall back on with answers and stuff, and what his body is going to be like in 5 years, because they don't know anything." (Participant 8)

\section{Genetics Follow-up}

Plans for future care and management were also explored. Although 12 of 14 parents indicated motivation to return for future follow-up in the genetics clinic, not all had an accurate understanding of the aspects of such a return visit. Some parents correctly recalled that the visit would include a physical and developmental evaluation in addition to determining whether new information about the VUS was available $(n=$ 4). Some assumed the doctor/genetic counselor would call whenever new information became available. Many discussed having their child "tested again;" some spoke specifically of future clinical tests (e.g., whole exome sequencing), while others discussed the possibility of repeating the CMA. A 
recurring misconception was the expectation that the genetics laboratory was holding a DNA sample that would be tested automatically as new technology became available:

"From what I understand, as new channels of testing become available, they're going to run her blood through that and test for those things. If anything pops up, then we're going to go back and kind of go from there." (Participant 7)

Many parents described confidence in their provider's expertise and expected the genetics team to play a future role in their child's health care $(n=5)$. The majority expressed an understanding that in time the significance and implication of the variant would likely be elucidated. Most were hopeful and optimistic that their questions would eventually be answered $(n=8)$; however, some worried that "bad news" about their child's prognosis would be forthcoming $(n=2)$. Most comments were framed with an expectation that the ambiguity surrounding the test result would be resolved $(n=8)$ :

"These tests are going to be just breaking through, left and right, in the next year at the latest. So, I'm really excited for that." (Participant 9)

"Maybe it will [explain the medical issues] down the line when they find out what it means to have the extra material on chromosome 8." (Participant 6)

\section{Discussion}

This study explored the experiences of families whose children had variants of uncertain significance identified through CMA analysis. The results provide answers to the very important questions raised by Fanos in her 2012 commentary: how well do parents understand microarray, genome, and exome sequencing results, and how do parents cope with the ambiguity and uncertainty of such results (Fanos 2012)? In our study, we discovered variability in several domains, including: parental recall and understanding; the impact of results on health, education, and therapeutic management; and parental interpretation. However, we also identified several emerging themes, including parental focus on personally meaningful rather than technical details, similarities in emotional responses to a VUS compared to a definitive diagnosis, and contradictions in personal results interpretation. Parents' intentions to comply with recommended follow-up were also investigated, and we noted strong motivation to do so, but with some misunderstanding of key components of this process. Our findings support those of previous studies which indicated incomplete comprehension and struggles with personal interpretation of results (Jez et al. 2015; Reiff et al.
2012), and further contribute to the literature surrounding patient understanding, interpretation and adaptation to genetic results of uncertain significance.

\section{Parental Recall of Information}

Many parents in our study struggled to describe the purpose of CMA analysis and could not recall what was being interrogated by the test. Several could not remember specific details about their child's VUS, although most were able to correctly recall whether a deletion or duplication of material was found. All parents could recount the uncertainty surrounding the CMA result, although the specific terminology (VUS) was rarely remembered. Factors contributing to uncertainty were also recalled, e.g., rarity and lack of clinical information. These findings support the premise that parents do not find information about the CMA analysis and the specifics of the variant identified particularly salient, and therefore do not retain it over time, despite the fact that these details are included in pretest counseling, informational materials, and a letter summarizing the results. Whitmarsh et al. (2007) suggested that even with time, additional information, and interactions with providers, a parent's understanding of the genetics of a diagnosis remains abstract. Furthermore, the use of technical language can contribute to confusion and misunderstanding, resulting in a negative experience (Ashtiani et al. 2014). This raises the essential question of what information is truly relevant to patients and families and how it is best presented. Technical details and specific terminology may best be avoided in favor of a more thorough discussion of broad concepts, allowing for greater emphasis on psychosocial issues (e.g., unmet expectations, emotional adjustment), a fundamental tenet of genetic counseling.

\section{Impact of Results}

Findings from our study suggest that receiving a VUS result may have an impact similar to that of receiving a definitive diagnosis; it was often viewed as an "answer," albeit a more complicated one. Parents described emotional responses ranging from strong feelings of guilt, sadness, and a sense of loss. Others described an evolution in their responses to that of comfort and relief. Some parents reported positive coping through engagement with support groups or advocacy activities. Others discussed that the test results empowered them to seek out medical, educational, therapeutic, and financial services. Similar benefits of receiving results were also noted in previous studies (Jez et al. 2015; Reiff et al. 2012; Whitmarsh et al. 2007). This suggests that even in the face of ambiguity, endorsing a parental perspective of active engagement with healthcare providers, teachers, and others, as well as accessing resources, can help to facilitate adaptation and coping. 
Uncertainty itself can have emotional consequences; parental anxiety, depression, and/or helplessness have been reported in parents when confronting the uncertain nature of their child's condition (Lipinski et al. 2006). In fact, some parents in our study reported difficulties with adapting to their child's result and engaged in negative coping such as pessimism, hopelessness, and feelings of failure. Lipinski et al. (2006) found that parents of children with chromosome abnormalities generally had high levels of uncertainty and felt that they had little control over their child's condition. We therefore propose that in many ways, parental reactions to receiving a VUS are even more complex and have the potential to be more emotionally charged with feelings of loss of control. Post-test genetic counseling for a VUS should acknowledge such responses and incorporate their possibility into the discussion of uncertainty to help facilitate parental coping. Results from our study and others suggest that encouraging parents to seek out services after receiving a VUS result may facilitate positive coping and promote feelings of control.

\section{Parental Interpretation of Results}

Similar to confronting a diagnostic result, parents in our study sought to personalize the meaning of the VUS for their child and family. According to Lazarus and Folkman's (1987) Transactional Model of Stress and Coping, this process involves an assessment of the impact a diagnosis will have on daily life, followed by consideration of what can be done (Lazarus and Folkman 1987). We found that many parents struggled with this adaptive process and postulate that it was in part due to the contradictory nature of their personal views of the results. Especially intriguing were the recurring opposing statements about parental interpretation of the VUS as an "answer" but not an "explanation" and vice versa. Furthermore, while some parents made statements indicating acknowledgment of uncertainty, they also asserted feelings of more certainty about pathogenicity, expressing that the VUS was "a point of reference" or "something to go on." This was also seen in the Vos et al. (2008) study in which patients who had a VUS in BRCA1/2 were more likely to personally interpret their results as pathogenic, even while correctly recalling that a VUS had been identified; Vos et al. questioned whether this was due to a disconnect between recall and perception or whether it was influenced by other beliefs.

We propose that it may be easier for some families to categorize ambiguous results in a more conclusive manner for purposes of coping and adaptation. In fact, the perception of permanence regarding results was described by some parents, indicating perhaps acceptance and/or the perception that a genetic variant was the equivalent of a diagnosis. Others expressed that their perceptions were instinctive or even based on hope, perhaps reflecting a strong desire for answers. A recent study reported that many parents viewed a VUS as having provided an explanation and therefore had experienced relief (Jez et al. 2015), a sentiment echoed by some participants in this study. Of course, the harm in this personal interpretation would be the possibility that families may not pursue additional follow-up evaluation, potentially preventing the establishment of a definitive diagnosis and/or the necessity for readaptation should an alternative diagnosis be made. In this study population, these types of categorizations did not seem to negatively impact the maintenance of a connection with the genetics team, as most all respondents clearly stated their intention to return for follow-up. For some, maintaining an effective separation between cognitive and emotional perceptions of variants of uncertain significance is feasible.

Reiff et al. (2012) found that parents held inconsistent views about their child's CMA result (whether a definitive pathogenic result or a VUS) supporting the perception it had helped them to know what to expect, while also acknowledging a lack of prognostic information. In our study, some of those who endorsed the perception that the VUS was not the cause of their child's medical problems also expressed incongruous feelings of personal validation. We suggest that, for these parents, the VUS confirmed their belief that something was different about their child, yet they could not characterize the VUS as causal due to the lack of clarifying and prognostic information.

Importantly, some parents made statements in support of the positive nature of uncertainty, voicing the idea that having a VUS made their child unique. Others found this isolating, reminiscent of the early experiences of families of children diagnosed with rare disorders described in the Fanos (2012) commentary. This is concerning given the likelihood of a growing frequency of ambiguities and difficulties with results interpretation in the era of advancing genetic technology. Clearly, individuals have varying tolerances toward uncertainty, some of which may relate to their views about genetic testing and/or healthcare, in general (Biesecker et al. 2014). This highlights the importance of pretest counseling that includes promoting a view of the evaluation process that is more open-ended rather than linked to a genetic test result. Whitmarsh et al. (2007) noted that amid the medical expertise associated with a genetic diagnosis, families who value their own experiences and adopt an open approach, emphasizing their child's individuality, are undertaking important processes that result in a valuing of the unknown. Including a discussion about the possibility of uncertainty in genetic testing and results - from the clinical significance of a CNV to the prognostic limitations of even well-defined syndromes - prior to testing, rather than focusing on an attempt to reduce uncertainty through diagnostic testing, may help parents form more realistic expectations regarding the outcomes of testing. 


\section{Genetics Follow-Up}

Previous studies of genetics follow-up have mostly focused on medical management for known genetic disorders, such as screening for cancer predisposition syndromes (Schneider and Schmidtke 2014). We were therefore particularly interested in examining parental responses to queries about their intention to comply with recommended follow-up in pediatric genetics in the setting of a VUS. Palmer et al. (2014) recently reported that a significant number of VUS identified in 2010 had been reclassified as "potentially pathogenic" or as a "susceptibility or modifier CNV" just 2 years later. We feel it imperative that genetics professionals emphasize the importance of a follow-up genetics evaluation to allow for a review of available databases, the literature, and a re-evaluation of the patient. Otherwise, families may be lost to follow-up and miss updated information that could impact their child's health care.

We found that, despite differing interpretations of the VUS as an answer but not as providing any clarification, the recommendation and importance of follow-up were accurately recalled, and all parents expressed intentions to do so. Interestingly, the majority of participants expressed the belief that the significance of the VUS would be elucidated and that eventually their questions would be answered. However, some parents were confused about the follow up process. Some believed that the geneticist/genetic counselor would automatically initiate contact once new information regarding their child's VUS became available. This confusion occurred despite explicit statements made during clinic visits and in summary letters recommending a return visit after a specified period of time (usually 1-2 years). Clearly, this highlights the need to further improve communication. We have addressed this in our clinic since this study by designing a brief patient/ family education sheet provided as a supplement to pretest counseling that includes a statement about the possibility of a VUS requiring follow-up and potentially further testing.

\section{Study Limitations}

This study has a number of limitations. Due to a limited number of eligible subjects, piloting of the interview guide was only possible on a small sample of pediatric genetic counselors. The study population was also limited to one medical center and subject to self-selection bias, and therefore the results are not generalizable to all populations undergoing CMA. The small sample size limited the ability to make comparisons between groups within the sample; e.g., established patients who had undergone pretest counseling versus those who had CMA ordered by an outside provider, VUS which were determined to be de novo versus inherited. Inconsistencies between and within these groups also included results disclosure, with some being contacted by phone by Pediatric
Genetics staff with variable amounts of information discussed prior to the in-person CMA counseling appointment, and others being told they would review all information at the time of their genetics appointment. Due to a desire to focus on issues related to genetic uncertainty and given limited resources, this study did not recruit parents of children who had clearly pathogenic or normal CMA results, resulting in the inability to make direct between-group comparisons. However, our study identified themes that appear to be similar to those expressed by parents receiving pathogenic results. We also acknowledge that retrospective attempts to elicit memory of genetic counseling sessions are subject to recall bias. Furthermore, the scope of our study did not allow us to determine whether participants had successfully completed follow-up per our recommendations.

\section{Conclusions}

Chromosomal microarray analysis is a powerful diagnostic tool, resulting in the identification of new syndromes and expanding understanding of the phenotypic spectrum of previously described disorders. However, interpretation of CMA results can be problematic, as there is a significant likelihood of identifying a copy number variant of uncertain significance. This presents challenges for parents who have been seeking a diagnosis for their child. Genetic providers need to address the lack of available information and help parents identify effective coping strategies. These challenges will only increase as genomic sequencing is implemented as a clinical diagnostic tool and sequence variants for which little or no information exists are identified. Furthermore, it is likely that the gap between identification and interpretation will grow in the foreseeable future as utilization increases due to decreasing costs of sequencing (Cutting 2014).

While the findings reported here were collected from a study of CMA results, they may also apply to other genomewide diagnostic tools currently being implemented in clinical genetics and other medical specialties which also have the potential to identify findings of uncertain clinical significance. Our results reinforce the value of pre- and post-test genetic counseling and suggest that results disclosure of a VUS should be similar in many ways to returning a definitive genetic test result. While communicating relevant information, it is critical to attend to emotional responses and explore how parents integrate genetic information into their view of their child's illness. In order to effectively facilitate parental coping and adaptation, genetics professionals should be prepared for a wide range of reactions and personal interpretations. Endorsing an open-minded approach to their child's future in the face of uncertainty may be particularly beneficial. Furthermore, it is important that parents clearly understand the importance of follow-up, and this may require multiple explicit reminders. 
Acknowledgments We thank the parents who participated in interviews as a part of this study. We also thank Alicia Giordimaina, Danielle Dubois, Bailey Hulswit, and Emily Wagoner for their assistance with this study. This work was supported by the National Society of Genetic Counselors Jane Engelberg Memorial Fellowship, the Michigan Association of Genetic Counselors, Inc., and the University of Michigan Rackham Graduate School. This study was completed in partial fulfillment of the requirements for Master of Science, University of Michigan Genetic Counseling Program, Ann Arbor, Michigan.

Conflict of Interest Authors Lesli A. Kiedrowski, Kailey M. Owens, Beverly M. Yashar, and Jane L. Schuette declare that they have no conflict of interest.

Human Studies and Informed Consent All procedures followed were in accordance with the ethical standards of the responsible committee on human experimentation (institutional and national) and with the Helsinki Declaration of 1975, as revised in 2000. Informed consent was obtained from all patients for being included in the study.

\section{References}

Ali-Khan, S. E., Daar, A. S., Shuman, C., Ray, P. N., \& Scherer, S. W. (2009). Whole genome scanning: resolving clinical diagnosis and management amidst complex data. Pediatric Research, 66(4), 357363. doi:10.1203/PDR.0b013e3181b0cbd8.

Ashtiani, S., Makela, N., Carrion, P., \& Austin, J. (2014). Parents' experiences of receiving their child's genetic diagnosis: A qualitative study to inform clinical genetics practice. American Journal of Medical Genetics Part A, 164(6), 1496-1502. doi:10.1002/ajmg.a.36525.

Biesecker, B. B., Klein, W., Lewis, K. L., Fisher, T. C., Wright, M. F., Biesecker, L. G., \& Han, P. K. (2014). How do research participants perceive "uncertainty" in genome sequencing? Genetics in Medicine: Official Journal of the American College of Medical Genetics, 16(12), 977-980. doi:10.1038/gim.2014.57.

Coughlin, C. R., Scharer, G. H., \& Shaikh, T. H. (2012). Clinical impact of copy number variation analysis using high-resolution microarray technologies: advantages, limitations and concerns. Genome Medicine, 4(10), 80. doi:10.1186/gm381.

Cutting, G. R. (2014). Annotating DNA variants is the next major goal for human genetics. American Journal of Human Genetics, 94(1), 5-10. doi:10.1016/j.ajhg.2013.12.008.

DeCuir-Gunby, J. T., Marshall, P. L., \& McCulloch, A. W. (2011). Developing and using a codebook for the analysis of interview data: an example from a professional development research project. Field Methods, 23(2), 136-155. doi:10.1177/1525822X10388468.

Fanos, J. H. (2012). New "first families": the psychosocial impact of new genetic technologies. Genetics in Medicine: Official Journal of the American College of Medical Genetics, 14(2), 189-190. doi:10. 1038/gim.2011.17.

Graungaard, A. H., \& Skov, L. (2007). Why do we need a diagnosis? A qualitative study of parents' experiences, coping and needs, when the newborn child is severely disabled. Child: Care, Health and Development, 33(3), 296-307. doi:10.1111/j.1365-2214.2006.00666.x.

Jez, S., Martin, M., South, S., Vanzo, R., \& Rothwell, E. (2015). Variants of unknown significance on chromosomal microarray analysis: parental perspectives. Journal of Community Genetics. doi:10.1007/ s12687-015-0218-4.

Lazarus, R. S., \& Folkman, S. (1987). Transactional theory and research on emotions and coping. European Journal of Personality, 1(3), 141-169. doi:10.1002/per.2410010304.
Lee, C., Iafrate, A. J., \& Brothman, A. R. (2007). Copy number variations and clinical cytogenetic diagnosis of constitutional disorders. Nature Genetics, 39(7 Suppl), S48-54. doi:10.1038/ng2092.

Lewis, C., Skirton, H., \& Jones, R. (2010). Living without a diagnosis: the parental experience. Genetic Testing and Molecular Biomarkers, 14(6), 807-815. doi:10.1089/gtmb.2010.0061.

Lipinski, S. E., Lipinski, M. J., Biesecker, L. G., \& Biesecker, B. B. (2006). Uncertainty and perceived personal control among parents of children with rare chromosome conditions: The role of genetic counseling. American Journal of Medical Genetics. Part C, Seminars in Medical Genetics, 142C(4), 232-240. doi:10.1002/ajmg.c.30107.

Lopez-Rangel, E., Mickelson, E. C. R., \& Lewis, M. E. S. (2008). The value of a genetic diagnosis for individuals with intellectual disabilities: optimising healthcare and function across the lifespan. The British Journal of Developmental Disabilities, 54(Part 2), 69-82.

Manning, M., Hudgins, L., Practice, P., \& Committee, G. (2010). Arraybased technology and recommendations for utilization in medical genetics practice for detection of chromosomal abnormalities. Genetics in Medicine: Official Journal of the American College of Medical Genetics, 12(11), 742-745. doi:10.1097/GIM. 0b013e3181f8baad.

Michie, S., Marteau, T. M., \& Bobrow, M. (1997). Genetic counselling: the psychological impact of meeting patients' expectations. Journal of Medical Genetics, 34(3), 237-241.

Miller, D. T., Adam, M. P., Aradhya, S., Biesecker, L. G., Brothman, A. R., Carter, N. P., \& Ledbetter, D. H. (2010a). Consensus statement: chromosomal microarray is a first-tier clinical diagnostic test for individuals with developmental disabilities or congenital anomalies. American Journal of Human Genetics, 86(5), 749-764. doi:10. 1016/j.ajhg.2010.04.006.

Miller, F. A., Hayeems, R. Z., \& Bytautas, J. P. (2010b). What is a meaningful result? disclosing the results of genomic research in autism to research participants. European Journal of Human Genetics: EJHG, 18(8), 867-871. doi:10.1038/ejhg.2010.34.

Palmer, E., Speirs, H., Taylor, P. J., Mullan, G., Turner, G., Einfeld, S., \& Mowat, D. (2014). Changing interpretation of chromosomal microarray over time in a community cohort with intellectual disability. American Journal of Medical Genetics Part A, 164A(2), 377-385. doi:10.1002/ajmg.a.36279.

Reiff, M., Bernhardt, B. A., Mulchandani, S., Soucier, D., Cornell, D., Pyeritz, R. E., \& Spinner, N. B. (2012). "What does it mean?": uncertainties in understanding results of chromosomal microarray testing. Genetics in Medicine: Official Journal of the American College of Medical Genetics, 14(2), 250-258. doi:10.1038/gim.2011.52.

Rosenthal, E. T., Biesecker, L. G., \& Biesecker, B. B. (2001). Parental attitudes toward a diagnosis in children with unidentified multiple congenital anomaly syndromes. American Journal of Medical Genetics, 103(2), 106-114.

Schneider, K. I., \& Schmidtke, J. (2014). Patient compliance based on genetic medicine: a literature review. Journal of Community Genetics, 5(1), 31-48. doi:10.1007/s12687-013-0160-2.

Shaffer, L. G., Coppinger, J., Alliman, S., Torchia, B. A., Theisen, A., Ballif, B. C., \& Bejjani, B. A. (2008). Comparison of microarray-based detection rates for cytogenetic abnormalities in prenatal and neonatal specimens. Prenatal Diagnosis, 28(9), 789-795. doi:10.1002/pd.2053.

Turbitt, E., Halliday, J. L., Amor, D. J., \& Metcalfe, S. A. (2014). Preferences for results from genomic microarrays: comparing parents and health care providers. Clinical Genetics. doi:10.1111/cge.12398.

Vos, J., Otten, W., van Asperen, C., Jansen, A., Menko, F., \& Tibben, A. (2008). The counsellees' view of an unclassified variant in BRCA1/ 2: Recall, interpretation, and impact on life. Psycho-Oncology, 17(8), 822-830. doi:10.1002/pon.1311.

Whitmarsh, I., Davis, A. M., Skinner, D., \& Bailey, D. B., Jr. (2007). A place for genetic uncertainty: parents valuing an unknown in the meaning of disease. Social Science \& Medicine (1982), 65(6), 1082-1093. doi:10.1016/j.socscimed.2007.04.034. 